\section{Contar para curar: estadísticas y comunidad médica en Argentina, 1880-1940*}

\section{Counting to cure: statistics and the medical community in Argentina, 1880-1940}

Claudia Daniel

Doctora en Ciencias Sociales por la Universidad de Buenos Aires. Chile 870 - Villa Sarmiento Haedo Provincia de Buenos Aires - Argentina

claudiadaniel@gmail.com

Recebido para publicação em outubro de 2010. Aprovado para publicação em junho de 2011.
DANIEL, Claudia. Contar para curar: estadísticas y comunidad médica en Argentina,1880-1940. História, Ciências, Saúde - Manguinhos, Rio de Janeiro, v.19, n.1, jan.-mar. 2012, p.89-114.

\section{Resumen}

Analiza los vínculos entre el grupo profesional médico y la

institucionalización de la estadística en la Argentina del período 1880-1940. Presenta los inicios de las estadísticas médicas y demográficas en el país, vinculados con los ideales y proyectos higienistas de fines del siglo XIX. Describe la posterior organización y estabilización de una agencia estatal encargada de producir esas cifras que tuvo la particularidad de ser dirigida por una mujer durante varias décadas. Indaga el problema planteado en relación con la formación de cuadros técnicos para la estadística pública. Explora las fuentes de tensión y las polémicas en torno al método numérico que se entablaron en la comunidad médica, reconstruidas a partir de las revistas especializadas.

Palabras clave: historia de la estadística; médicos; Sección Demografía y Geografía Médica; Argentina.

\section{Abstract}

The article analyzes the links between the professional medical community and the institutionalization of statistics in Argentina in 1880-1940. It begins with the initial period of medical and demographic statistics in Argentina, which bore ties to late nineteenth-century hygienist ideals and projects. It then describes the later organization and consolidation of a state agency responsible for producing these data, which, interestingly enough, was headed by a woman for several decades. The

challenges encountered in training technical staff to produce public statistics are also examined. Based on information found in specialized magazines, the analysis likewise explores the tensions and controversies that arose within the medical community over the use of the numerical method.

Keywords: history of statistics; medical statistics; Demography and Medical Geography Section; Argentina. 
$\mathrm{E}$ el transcurso del siglo XIX, los médicos cobraron un rol preponderante en la constitución de los campos estadísticos en distintas naciones de Europa y América. Para ello, impulsaron investigaciones, crearon instituciones especializadas y motorizaron proyectos editoriales que dieron publicidad a esas cifras. En México, los médicos higienistas participaron activamente del establecimiento de rutinas de recuento e influyeron en la realización de censos (Cházaro, 2010; Mayer Celis, 1999). En Brasil, los demógrafos sanitaristas protagonizaron interesantes polémicas con los números oficiales y algunos de sus representantes alcanzaron posiciones gravitantes en el órgano principal de la estadística nacional (Camargo, 2007; Senra, 2009). En la Argentina, un grupo de profesionales médicos se vio involucrado en los inicios de los estudios sociales de carácter cuantitativo durante la segunda mitad del siglo XIX. ${ }^{1}$ Abocados a buscar soluciones a los graves problemas de insalubridad de las ciudades (epidemias, hacinamiento, etc.), esos médicos buscaron asentar, en los conocimientos creados mediante herramientas cuantitativas, las bases de una política científica para enfrentar dichas cuestiones.

Los médicos higienistas, preocupados con la salud del 'cuerpo social', se inclinaron a dotar al Estado de capacidades de recolección de información y a tratar de organizar rutinas estables de recuento en sus agencias. Estos profesionales supieron capitalizar su cercanía a los centros de poder local, ocuparon crecientemente posiciones en el Estado durante el orden político conservador y expandieron los márgenes de su área de acción construyendo socialmente (la necesidad de) un entramado institucional que se enfocara a los problemas de salubridad de las principales ciudades.

La historiografía argentina se ha ocupado de analizar en profundidad las prácticas de intervención en la trama social y urbana, desplegadas por los médicos entre la segunda mitad del siglo XIX y las primeras décadas del XX. La contribución de estos médicos fue estudiada desde el punto de vista de la creación de instituciones especializadas en salud pública, su participación en la definición de las políticas estatales y en el establecimiento de una agenda política, al convertir a la enfermedad en un problema social. (Armus, 1984, 2000b; Armus, Belmartino, 2001; González Leandri, 2004; Salvatore, 2001) Desde un enfoque histórico-social, se observó cómo el higienismo se fue transformando paulatinamente en un código moral que permeó la sociedad argentina (Armus, 2000a, 2000b). Desde el punto de vista de una sociología histórica de las profesiones, también fue estudiado el proceso específico de profesionalización médica en Buenos Aires, teniendo en cuenta las pujas por la delimitación de fronteras jurisdiccionales y la competencia con otros saberes; proceso en el que la promoción de una nueva concepción de la salud operó legitimando la ampliación de dichos márgenes (González Leandri, 2000).

Teniendo en cuenta estos importantes aportes, nos interesa plantear una serie de interrogantes que se muestran transversales a las dimensiones ya estudiadas (la estatal, la social y la profesional), pudiendo echar luz sobre nuevos aspectos de estos procesos sociales ¿De qué manera se enlazó la práctica social y política de los médicos y las médicas en el Estado con la expansión de la racionalidad estadística en la Argentina? ¿Qué clase de vínculos ligaron la comunidad médica con la burocracia técnica especializada en la producción de las cifras oficiales? ¿Cuáles fueron las reacciones que despertaron los números públicos entre los miembros de este campo disciplinario? El objetivo de este trabajo es 
conocer la gravitación que alcanzó la comunidad profesional médica en el proceso de institucionalización de la práctica estadística en la Argentina, así como explorar las repercusiones que tuvo la generalización de las herramientas estadísticas en la propia configuración del campo médico.

La sociología y la historia del conocimiento estadístico es un área de investigación en crecimiento en los últimos años. En la Argentina, los aportes iniciales a este terreno adquirieron la forma de estudios sobre la actividad estadística oficial, los modos de organización burocrática y su normativa en articulación con los contextos económicos y políticos de formulación (Daniel, 2009; Indec, 1983; Massé, 2000; Mentz, 1991; Novick, 2002). La historia institucional de la estadística pública tomó un nuevo giro gracias a la reconstrucción de la trayectoria social y política de una elite burocrática especializada y sus efectos en las políticas estatales (González Bollo, 2007). Jorge Pantaleón (2009) analizó la contribución de las estadísticas en la construcción del espacio nacional y el surgimiento de la planificación estatal en la Argentina del período 1918-1952. El historiador Hernán Otero (2006) inauguró el estudio de los conceptos y las categorías de análisis presentes en las herramientas de cuantificación (censos, encuestas, etc.), utilizadas por la estadística argentina decimonónica al trasluz de las influencias científicas, políticas e ideológicas a las que estuvo sujeta. En un trabajo reciente, se exploró el proceso histórico de construcción de la autoridad social de las estadísticas entre 1890 y 1940 (Daniel, 2010). Sin embargo, fuera del caso del campo de la economía - cuya conformación fue asociada con la estadística (Pantaleón, 2009) - los vínculos entre el desarrollo de una profesión en particular y las prácticas estadísticas no fueron objeto de análisis específicos. A excepción del estudio sobre las estadísticas de salud en los Territorios Nacionales de la historiadora Silvia Di Liscia (2009), parecería ser que las propias limitaciones de la estadística médica y demográfica argentina confinaron a este objeto de estudio a un lugar de escasa visibilidad en el terreno de la historiografía estadística nacional.

El período temporal de este trabajo se inicia en 1880 por varias razones. Primero, la creación del Departamento Nacional de Higiene (DNH) expresa - en el proceso histórico de conformación del Estado argentino - una mayor complejidad de su estructura administrativa. Dentro de este espacio burocrático, los alcances o limitaciones de las tareas de recopilación de datos demográficos o sanitarios - y, en general, el éxito o el fracaso del Estado en la concentración de un tipo de capital fundamental para el ejercicio de la dominación como la información - nos permiten problematizar el proceso de penetración estatal en el territorio nacional. A su vez, en la década de 1880, se ponen en marcha diversas iniciativas de recopilación de estadísticas demográficas, esfuerzos locales que guardaban aspiraciones más amplias y la voluntad de los especialistas de transformar las actividades aisladas de recuento en una tarea regular del Estado. Se trata de una década prolífera en censos. ${ }^{2}$ El marco temporal escogido se extiende hasta el ocaso de la trayectoria como funcionaria, sanitarista y demógrafa de la doctora Adela Zauchinger que estuvo al frente de la repartición estadística del DNH durante tres décadas. Los cambios introducidos en la estructura del Estado tras el golpe militar de 1943 la encuentran fuera del organigrama estatal. El gobierno de facto crea la División de Bioestadística y Geografía Médico Social junto a la Dirección Nacional de Salud Pública bajo la órbita del Ministerio del Interior. Se 
abre en la Argentina una etapa de inestabilidad, no sólo política sino de avances y retrocesos de modelos organizativos, de división y unificación de organismos estadísticos, que sería objeto de otra investigación.

Este trabajo parte de la idea de que en la Argentina los médicos fueron protagonistas del desembarco de la racionalidad estadística en las últimas décadas del siglo XIX. Sin embargo, esa voluntad precursora no alcanzó para asegurarles una posición dominante en el proceso de institucionalización de la práctica estadística. La colonización, incluso de un espacio propio dentro del aparato estadístico nacional - con la suficiente continuidad en el tiempo -, no le garantizó a este grupo profesional una posición gravitante en el campo estadístico en constitución. Esto estuvo relacionado con el hecho de que la expansión de la racionalidad estadística en el campo médico se vio frenada por el desinterés mostrado por la comunidad profesional respecto de la formación de cuadros especializados en la aplicación de las herramientas cuantitativas y por las dificultades en alcanzar un consenso sólido alrededor del método numérico.

\section{Los médicos en el aparato estadístico oficial}

\section{Los precursores}

Durante las últimas décadas del siglo XIX, el proceso de modernización económica y social que experimentó la Argentina se caracterizó por el afianzamiento de una economía agroexportadora, por la recepción de una inmigración masiva y por un crecimiento urbano acelerado. Como consecuencia de esos procesos, en las más grandes ciudades surgieron problemas de insalubridad, hacinamiento, pobreza y diversas formas de marginalidad. En este contexto, la salud de la población se estableció como uno de los principales problemas sociales que preocuparon a la elite política. Entonces, los médicos, como poseedores de un saber especializado y oficialmente acreditado, empezaron a ocupar posiciones en el Estado. ${ }^{3}$ Desde ese lugar, impulsaron intervenciones públicas sobre la trama urbana (como las obras de salubridad), organizaron un dispositivo institucional para hacer efectivo el gobierno sanitario de la sociedad (con la creación del DNH y de la Administración Sanitaria y Asistencia Pública de Buenos Aires) e impulsaron una agenda higienista con el propósito de preservar y fortalecer la salud de la población.

Desde su posición en el entramado burocrático estatal, algunos médicos higienistas introdujeron los estudios estadísticos en el país como instrumento de una política científica que tenía por objeto el bienestar de la sociedad. Esa política no sólo se orientaba a asegurar el crecimiento demográfico sino también a construir una comunidad nacional moderna, sana y armónica (es decir, libre de conflictos). El doctor Emilio R. Coni (1854-1928), desde la Dirección General de Estadística de la provincia de Buenos Aires (que dirigió entre 18831886), bregó por la organización de rutinas sistemáticas de recopilación de información sobre la población como base del estudio de los principales problemas que la afectaban. ${ }^{4}$ La importancia reconocida a las herramientas estadísticas ante estas cuestiones no fue tanto un legado de su formación universitaria sino un descubrimiento temprano. Coni se inició, a los 20 años, como delegado de esa Oficina de Estadística (1874-1880), aún sin estar recibido de médico. En 1881, fue secretario de la Comisión del Censo General de la provincia 
de Buenos Aires. ${ }^{5}$ En 1884, fue director del Censo de La Plata y de distintos partidos bonaerenses. A través de sus distintos viajes a Europa, Coni entabló relaciones con las reparticiones estadísticas de varios países y con algunos de los principales referentes de la estadística internacional. Esos viajes le sirvieron para promover el intercambio de publicaciones pero también para importar modelos organizativos (división de tareas, organización del personal, reglamentos) y operativos (libretas, registros, grillas, clasi-ficaciones) para la estadística local.

Algunos médicos se abrieron camino en la burocracia pública y en la orientación de las políticas sociales portando ese 'saber hacer' estadístico. El manejo de información cuantitativa y su versación en técnicas modernas tan valoradas - en el país como en el exterior -, sumado al prestigio que ya había alcanzado la profesión médica, les sirvieron para afirmarse públicamente como los proveedores legítimos de soluciones específicas ante los problemas de salud de la población. Así, en la Argentina finisecular, los médicos lograron cierto dominio de las instituciones reformistas y de las políticas de regulación de los sectores populares.

Sin embargo, en las últimas décadas del siglo XIX, el quehacer estadístico se ubicaba todavía en un terreno difuso. La divisoria entre investigaciones privadas y gubernamentales permanecía borrosa. Ello significaba la existencia de publicaciones oficiales de datos sostenidas con recursos particulares; o viceversa, capitales del Estado invertidos en trabajos presentados en congresos o asociaciones científicas a título personal. En un período de formación y consolidación del aparato estatal, pero también del colectivo profesional médico, los tránsitos pendulares entre el ejercicio privado de la profesión, el campo académico y el Estado eran asiduos. Las investigaciones estadísticas provenían fundamentalmente de esfuerzos individuales ya que todavía resultaba difícil estabilizar rutinas sistemáticas de recuento. Por tanto, la labor estadística discurría en un terreno disipado entre la tarea oficial en las oficinas públicas, la formación profesional y la carrera académica, o la consecución de simples iniciativas personales. ${ }^{6}$ Como director de la Oficina Estadística de la provincia de Buenos Aires, Coni presentó trabajos estadísticos en el Congreso Internacional de Higiene, reunido en La Haya en 1884. Asimismo, cuando la estrechez del presupuesto público afectó la divulgación de las cifras oficiales, no dudó en sostener financieramente la publicación de los primeros boletines de estadística con sus recursos particulares.

El solapamiento entre lo público y lo privado que mostró la actividad estadística no constituyó una particularidad del caso argentino. En su análisis sobre la investigación médica en Inglaterra, en el marco del llamado movimiento estadístico de los años 18301840, Lacey y Furner (1993, p.105) señalan también la ausencia de una división clara entre investigaciones gubernamentales y extra-gubernamentales, entre 'profesionales' empleados por el Estado y 'amateurs' investigando por su propia cuenta. Estos autores observaron que tanto el gobierno como las sociedades letradas inglesas recurrían a los estudios estadísticos, independientemente de si se trataba de investigaciones oficiales o no. En Inglaterra, la comunidad de expertos estadísticos se fue conformando tanto dentro como fuera de la burocracia estatal. Los expertos ingleses constituyeron un 'servicio civil no oficial', disponible para (y cooptado por) el Estado cuando ese expertise se hacía necesario. 
Pero, si en Inglaterra los lazos de los estadísticos con la maquinaria gubernamental fueron más que nada informales y ad hoc, los especialistas argentinos asumieron una mayor dependencia respecto del Estado; ocupar posiciones en la burocracia pública les resultó fundamental para desplegar su actividad y les brindó el respaldo necesario para adquirir visibilidad pública. ${ }^{7}$

A fines del siglo XIX, más allá del campo médico, las enfermedades habían logrado concitar la atención y el interés de políticos, filántropos, urbanistas e intelectuales (Armus, 2000b). El carácter público del problema se encontraba ligado al nivel colectivo en el que eran ubicadas conceptualmente las enfermedades, en especial, las infectocontagiosas. A través del discurso médico, la enfermedad era configurada como una entidad independiente del enfermo particular que tomaba como blanco al 'cuerpo social'. La sociedad se concebía como un organismo amenazado por los diversos peligros que a su salud imponía la propia trama física y social de la urbe. La salud colectiva era percibida como un estado acechado permanentemente por el contagio. De allí la importancia de una vigilancia estadística constante. Las estadísticas periódicas emergían como el instrumento capaz de tomarle el pulso a la sociedad, de medir la vitalidad del 'cuerpo social'. El crecimiento demográfico era considerado uno de sus principales índices vitales. La reproducción cuantitativa de la población era entendida como una necesidad nacional.

Refiriéndose a su trayectoria profesional, el doctor Coni se reconoció públicamente como un médico de ciudades y de pueblos, antes que de enfermos. El carácter social de la enfermedad hacía necesaria una herramienta de agregación para su análisis; un instrumento técnico que 'distanciara' al médico del caso clínico individual y le posibilitara 'mirar socialmente' al enfermo. Los censos de población también se propusieron como una fotografía (o radiografía) del estado de salud del 'cuerpo social', señalando, a veces, las ventajas de las condiciones higiénicas y lo saludable del clima del país u, otras, advirtiendo a los poderes públicos acerca de las deficiencias de su estado sanitario. ${ }^{8}$ El censo provincial de Córdoba (realizado en 1890 bajo la dirección de Alberto Martínez) fue el primero en introducir la pregunta relativa al estado de salud de la población. El segundo censo nacional de 1895 - en el que Coni colaboró - también cuantificó a los enfermos. ${ }^{9}$ Resulta llamativo que entre las pocas preguntas que, según la comisión censal, la experiencia y el saber técnicos de la época aconsejaban realizar en este tipo de relevamientos masivos, la cuestión de la salud de la población asumiera suficiente relevancia para figurar entre ellas. Los enfermos eran registrados en el apartado denominado condiciones especiales de la ficha censal (junto a sordomudos, ciegos, idiotas, alienados, inválidos y con bocio o coto). Allí fue catalogada una población que, desde el punto de vista de los estadísticos, constituía una 'desventaja' para el progreso de la Nación. La comisión censal definió a los enfermos como personas afectadas de alguna dolencia física o psíquica que los inhabilitaba para el trabajo, al menos temporalmente. Su reconocimiento por parte del censista quedaba supeditado a que éste lo encontrara en cama al momento de la visita. La importancia de esta población numéricamente irrisoria era que contribuía a engrosar ese contingente social demarcado por la estadística pública como los ciudadanos considerados pasibles de asistencia. Sobre ese grupo, los censistas depositaban la responsabilidad social del Estado; le reconocían la obligación de tomarlos a su cargo por constituir los elementos pasivos de la sociedad. 
Los cuadros estadísticos del informe censal de 1897 muestran que, tanto en este como en otros aspectos relativos a la salubridad de la población, el interés de los censistas se dirigió hacia la observación de la distribución de enfermos según su carácter nacional o extranjero, antes que por su edad o su condición de género. A partir de ello, desde el 'paradigma censal' (Otero, 2006) se construía una representación del inmigrante como el adulto sano, fuerte y vigoroso, por contraposición al argentino, débil y enfermo. Esta imagen se correspondía con la objetivación estadística producida entonces por la oficina de la ciudad capital: el inmigrante vital y enérgico se postulaba como un elemento de prosperidad nacional. Ambas representaciones estadísticas depositaban en ese factor externo la construcción de una 'raza argentina' fuerte y sana, mientras contribuían a la legitimación del proyecto migratorio del Estado. Así lo hicieron hasta el cambio de siglo, cuando las voces desencantadas con el programa inmigracionista argentino empezaron a encontrar eco en las páginas de las publicaciones de la estadística municipal.

A partir de la década de 1890, las estadísticas regulares producidas por la Dirección General de Estadística Municipal de la Ciudad de Buenos Aires (DGEM) contribuyeron a jerarquizar ciertos temas dentro del complejo salud-enfermedad. Los índices de las sucesivas publicaciones de su Boletín Mensual (BMEM) bastan para observar cómo sus cifras actuaron destacando, en cada momento, los distintos factores que se consideraban de mayor importancia en cuanto afectaban la salud del organismo social. Estos factores iban desde afecciones físicas (sobre todo las enfermedades infecciosas como sarampión, viruela, escarlatina, difteria, fiebre tifoidea, tuberculosis) a las consideradas enfermedades sociales (como la prostitución, el alcoholismo o el juego). Desde este lugar, las estadísticas se instalaron como propulsoras de los múltiples dominios de la agenda higienista en la ciudad capital.

La oficina municipal de recuentos fue una de las tantas instituciones que Coni contribuyó a poner en marcha en $1887^{10}$ (Coni, 1918, p.114-117). A cargo de Alberto Martínez a partir de 1890 , sus boletines estadísticos conformaron ese corpus de conocimientos que decantaría en la definición de una política social considerada científica, "suministrando una base sólida a la profilaxia administrativa" (Oficina de Estadística..., 1887, p.33) ${ }^{11}$ Las estadísticas operaban dando entidad pública a ciertas enfermedades. Al mismo tiempo, esas cifras justificaban y autorizaban - desde su estatuto científico - la intervención estatal sobre algunos aspectos de las prácticas sociales. La herramienta cuantitativa actuaba reforzando la legitimidad de la agenda de intervención propuesta por el higienismo; una agenda que se orientó tanto a modificar la trama urbana como a reformar los comportamientos de ciertos sectores de su población.

Asimismo, las estadísticas vitales se instituyeron como vara de medición de los progresos realizados en materia higiénica. Los índices de morbilidad fueron utilizados como indicadores de los efectos positivos de las obras de salubridad en la población porteña. ${ }^{12} \mathrm{En}$ relación a su influencia en la disminución de ciertas enfermedades y en la caída de la mortalidad, el abogado Gabriel Carrasco - director de la Oficina Demográfica Nacional (1899-1906) - llevaba adelante el cálculo del número de vidas salvadas, 'ahorradas' según sus palabras, como consecuencia de dichas políticas. ${ }^{13}$ El vocablo elegido por Carrasco es representativo de la matriz conceptual con que era pensada la población, en la que el número de habitantes se concebía como reflejo del potencial económico de la Nación. 
Con los años, estas oficinas contaron con series estadísticas, que mostraban la evolución de la incidencia de las enfermedades en la población, y compartieron tecnologías. La DGEM perfeccionó la forma de clasificación de las causas de muerte y desarrolló planos de distribución topográfica de las defunciones ocasionadas por distintas enfermedades; ambas técnicas fueron adoptadas por la oficina demográfica nacional. La estadística pública incorporaba así una clasificación internacional de las enfermedades que había sido propuesta por el médico francés Jacques Bertillón (1851-1922) en 1893 ante el congreso del Instituto Internacional de Estadística realizado en Chicago (Desrosières, 1996). De esta manera, los titulares de las agencias locales buscaban ajustar su práctica de recuento a una convención de codificación que detentaba la autoridad de haber sido discutida y respaldada por la principal institución de la estadística mundial, refrendada más tarde en congresos médicos. La clasificación Bertillón fue promovida con miras a armonizar los datos estadísticos más allá de las fronteras nacionales. En Argentina, dicha clasificación se estableció como el patrón de definición de las categorías sobre las que basar la acción pública en materia preventiva y sanitaria. Para ello, la codificación del acta de defunción debía convertirse en un procedimiento estandarizado y burocrático, es decir, atento a un formulario prefijado. Un primer obstáculo radicaba en que, en varios lugares del país, estas actas no eran completadas por médicos, sino por personal burocrático no calificado. Sin embargo, los médicos podían llegar a plantear resistencias a ajustar su criterio profesional a esa codificación. La aplicación de la clasificación constreñía al médico a reducir la diversidad de los casos que encontraba a clases que habían sido construidas del otro lado del Atlántico. Ambos factores hacían que fuera todo un desafío volver esta clasificación realmente operativa en la amplia extensión del territorio argentino.

Por otro lado, el movimiento higienista y los congresos médicos de principios de siglo XX impulsaron la necesidad de contar con la expresión geoestadística de los fenómenos que eran de su principal interés. Para 1912, las estadísticas eran consideradas un insumo fundamental para "confeccionar un mapa sanitario del territorio, que indicara los focos y pequeños focos de epidemias comunes ... para atacarlos inmediatamente, circunscribirlos y limitar su acción" (Argentina, 1912, p.1076). La expresión de los fenómenos vitales y demográficos en el territorio a través de diagramas permitía recuperar - en una sola mirada - a ese organismo social 'amenazado' como un todo, identificar regiones o grupos sociales más vulnerables, atribuir causas constantes a las enfermedades y reconocer los factores que facilitaban su difusión. Contar enfermos formaba parte del registro continuo de los 'peligros' que acechaban al 'cuerpo social' promovido por el higienismo, observación permanente de los individuos (sanos e insanos) que era ejercida también en otros ámbitos de su práctica social como las inspecciones de higiene o los espacios de segregación y encierro (Salvatore, 2001). Así, el proyecto higienista, que articuló objetivos preventivos y de control social, se asoció con un lenguaje cuantitativo de comprensión de la vida social.

\section{Una mujer al frente de la estadística médica y demográfica}

Si bien Coni dio un importante impulso a la actividad estadística, dejando todo un legado a nivel institucional y editorial, apenas iniciado el siglo XX no encontramos ningún médico entre los directivos de las distintas agencias del aparato estadístico nacional. 
Tampoco los habría dentro del grupo que creó en 1937 la Sociedad Argentina de Estadística desde un ámbito extra-gubernamental como el Museo Social Argentino (MSA). ${ }^{14}$ Sin embargo, durante este lapso, cierto grupo de médicos seguiría vinculado a la producción estadística local en lo que podemos considerar como un espacio de refugio dentro del entramado burocrático estatal: la Sección Demografía y Geografía Médica (SDGM), creada en 1911 en el DNH.

Organizada por su director, el doctor José Penna (1855-1919) - reconocido higienista a cargo del DNH entre 1910 y 1916, quien fuera compañero de Coni en la primera comisión directiva de la Sociedad Médica Argentina (1891) -, esta agencia fue inicialmente creada como una oficina de epidemiología e informes sanitarios que buscaba reunir datos sobre el estado sanitario argentino con miras a mejorar las condiciones de supervivencia y reproducción de la población. Con su implantación, el DNH buscaba "ofrecer al país una estadística completa de las enfermedades infecto-contagiosas que lo afligen con cifras exactas, conocimiento indispensable para emprender una tarea de profilaxis útil, atacando el mal en las regiones más infectadas y necesitadas" (Argentina, 1911, p.143). Esta repartición quedó a cargo de la doctora Adela Zauchinger, única mujer del grupo local de expertos estadígrafos.

Adela Zauchinger nació en Francia el 6 de diciembre de 1883. Con 19 años, se inscribió en la Facultad de Medicina de Buenos Aires (1903), pidiendo ser eximida de derechos universitarios por no contar con recursos para su pago. Su ingreso a la carrera de medicina no sólo expresa un desafío a modelos sociales arraigados en la sociedad porteña de entonces, sino también supone de su parte el aprovechamiento estratégico de las conexiones y de los circuitos sociales disponibles para que una mujer accediera a una formación universitaria. ${ }^{15}$ Recibida en 1910, Zauchinger integró el grupo de 11 mujeres que se graduó en esa casa de estudio en la primera década del siglo XX. Ellas representaban el 1\% del total de diplomados (Kohn Loncarica, Sánchez, 2000).

Asimismo, Zauchinger formó parte de la primera generación de mujeres médicas que participó formalmente en el ámbito científico-institucional argentino. ${ }^{16} \mathrm{Su}$ ingreso a la función pública se concretó a través de uno de los mecanismos de reclutamiento del personal técnico del Estado más usuales de la época. Los contactos personales y, especialmente, los nexos establecidos con profesores universitarios que trabajaban en algunas de esas reparticiones públicas resultaban fundamentales para quienes, por su origen social, no tenían acceso directo a funcionarios gubernamentales. El ingreso de Zauchinger al DNH (1913) estuvo vinculado al padrinazgo de una figura importante en su campo disciplinar: el doctor Penna, quien había sido su profesor en la materia clínica epidemiológica y entonces era director del DNH. ${ }^{17}$ Penna dirigió la tesis con que ella se doctoró sobre La protección de la primera infancia. Este trabajo reconocía, como antecedente, el estudio comparativo realizado por Coni en 1892 donde alertaba a los poderes públicos sobre el problema de la mortalidad infantil. Esta especialización temática marcaría la orientación de las estadísticas recolectadas por la agencia estatal bajo el mando de Zauchinger.

La trayectoria social de esta médica refleja los roles entonces establecidos para la mujer en el ejercicio (privado y público) de la profesión: una vocación orientada a las áreas ligadas a la mujer o al niño y un ejercicio de la profesión subordinado a sus colegas 
masculinos. ${ }^{18}$ Su trabajo no quebró los márgenes de los dominios que le eran socialmente asignados a las médicas; límites apoyados en el reconocimiento de ciertos atributos y aptitudes consideradas naturales en la mujer en el imaginario de la época. Muchos de sus estudios estadísticos se publicaron con su firma junto a la de colegas masculinos, como los doctores Barbieri y Sussini, o subordinada a la del doctor Aráoz Alfaro, en su rol superior como director del $\mathrm{DNH}$.

Zauchinger estuvo al frente de la sección de estadísticas del DNH hasta 1940. Su producción era divulgada a través de una publicación institucional, el Anuario Demográfico. Parte de la labor de la SDGM consistía en reunir y concentrar los datos sobre natalidad, mortalidad y nupcialidad enviados desde los registros civiles del interior y de los Territorios Nacionales. Durante un tiempo, las capacidades de recolección de la SDGM descansaron en un cuerpo de corresponsales en las provincias y los Territorios Nacionales. Sin embargo, por problemas presupuestarios, estos corresponsales no tuvieron continuidad, minando las posibilidades de la oficina de alcanzar una producción estadística abarcadora del total del país. ${ }^{19}$ La composición de un retrato numérico completo del territorio nacional se vio obstaculizada por las resistencias a la centralización que no sólo la SDGM encontraba en los ámbitos de administración local sino que padecía el propio DNH frente a sus políticas. En este sentido, se subrayaba en las memorias del organismo que la realización del programa de la institución requería "del conocimiento previo de nuestra geografía médica y de ... las circunstancias propias a cada localidad, susceptibles de influir desfavorablemente en su grado de salubridad" (Argentina, 1911, p.5). Era para llegar a aprehender esta particularidad de lo local - y al mismo tiempo componer cuadros regionales - que la institución solicitaba encarecidamente la cooperación de las autoridades de las distintas jurisdicciones del país. Este interés escudriñaba objetivos centralizadores en la medida que las autoridades públicas pretendían llevar adelante una gestión nacional de los problemas de salud y avanzar así con el proceso de medicalización de la población.

Durante la década de 1920, el poder político se encontró frente a la necesidad de ampliar la autoridad estatal en materia sanitaria por medio de la consolidación de una organización central que coordinara políticas en todo el territorio y que contara con el presupuesto adecuado para aplicar esas medidas. En este marco, la estadística aparecía como la herramienta capaz de hacer efectiva una dimensión clave de esa tarea de centralización: la unificación cognitiva del territorio. Las estadísticas de la SDGM se habían vuelto un instrumento fundamental en la configuración de un espacio nacional, siendo partícipes de un esfuerzo de centralización política y administrativa que no llegó a afianzarse.

Durante el período de los gobiernos radicales (1916-1930), el plantel de la SDGM se mantuvo entre cinco y siete empleados, bajo el mando de una misma directora. En la misma época, otras agencias estadísticas como las existentes en el Ministerio de Hacienda (Dirección General de Estadística Nacional) y en la cartera de Agricultura (Dirección de Economía Rural y Estadística) contaban con una cantidad mucho mayor de recursos humanos; su plantel alcanzó los 50 empleados. ${ }^{20}$ Pese a lo limitado de sus recursos, los trabajos realizados por la oficina de Zauchinger fueron reconocidos por voces representativas de la comunidad médica y por publicaciones especializadas en la disciplina. ${ }^{21}$ Sin embargo, durante sus casi tres décadas de funcionamiento, la SDGM no logró tener más que un rol 
marginal dentro del aparato estadístico nacional. Algunos de los titulares de otras reparticiones estadísticas fueron muy críticos respecto del grado de evolución alcanzado por la estadística demográfica argentina, calificaron la tarea de Zauchinger como rudimentaria o pobre, e incluso emprendieron gestiones administrativas para quitarlas de su jurisdicción. Raúl Prebisch, desde su cargo de subdirector de la Dirección General de Estadística Nacional, impulsó la transferencia de las estadísticas demográficas a la repartición en la que él se desempeñaba. Se dirigió al director del DNH buscando la aprobación del traslado, pero el doctor Gregorio Aráoz Alfaro no cedió ante esas presiones. ${ }^{22}$

Aún cuando conservó cierta capacidad de resistencia, la SDGM ocupó una posición marginal en el entramado burocrático estatal. Esta marginalidad puede ser atribuida a distintos factores. Por un lado, la limitada organización de oficinas de estadística y de registros civiles en el interior del país mermaba las capacidades de recolección de datos de la repartición central. Al mismo tiempo, la persistencia de recelos a la entrega de información en ciertas dependencias burocráticas, tanto provinciales como municipales, se conjugaban con las escasas facultades de coordinación demostradas por la repartición de Zauchinger. Además, lo exiguo de sus recursos financieros y la negativa del Congreso a incrementarlos le significaron años sin poder editar su publicación institucional. ${ }^{23}$

Por otra parte, no podemos dejar de destacar el hecho de que la dirección de esta oficina haya recaído en manos de una mujer en el marco de una cultura predominantemente masculina - donde los espacios de ejercicio del poder, en los distintos campos, le eran reservados a los hombres y las posiciones de peso en la administración del aparato del Estado también. En este sentido, puede interpretarse que la dirección de una mujer tornaba doblemente débil a este reducto burocrático, al agudizar su problema de autoridad frente a otras reparticiones públicas. Las reticencias de sus responsables a cumplir con los pedidos de información (experimentados como avances sobre la autonomía local) posiblemente se acentuaran ante la situación de verse subordinados a los designios de una mujer. Podemos presumir también que en su condición de género recayera parte del fundamento de la marginación y la dureza de la crítica a la que se encontraba sujeta entre sus pares de la administración pública. No disponemos de elementos taxativos que confirmen o desacrediten estas hipótesis. Pero consideramos que ello no los invalida como factores a tener en cuenta en el análisis de la evolución de una repartición estatal de las características de la SDGM. ${ }^{24}$

Por último, un indicador adicional del carácter marginal de esta repartición se expresó más tarde en la duplicación de estructuras burocráticas al interior mismo del DNH. Tras la creación en 1936 de la Dirección de Maternidad e Infancia (ley 12.341), se reglamentó el funcionamiento de una oficina recolectora de datos que, por lo menos en los fines proclamados en la normativa, replicaba parte de las atribuciones de la SDGM. El decreto de 1937, firmado por los responsables de esa dirección, Aníbal Olarán Chans y Luis Siri, le otorgaba a una de sus divisiones - la de Higiene Social de la Infancia - la responsabilidad de hacer investigaciones demográficas, estadísticas sanitarias y estudios de todos los problemas de orden médico, higiénico y social relativos al niño (Biernat, Ramacciotti, 2008). ${ }^{25}$ Este solapamiento suponía negarle reconocimiento a lo realizado hasta el momento por la SDGM. 
La superposición de jurisdicciones señalada resulta relevante en la medida en que la oficina liderada por Zauchinger se caracterizó por una producción estadística orientada a la población infantil. Entre sus intereses cognoscitivos prioritarios estaban el cálculo de los índices de mortalidad infantil ${ }^{26}$ y el recuento de los nacimientos ilegítimos. El censo nacional de 1869 había inaugurado en el país la distinción, al interior de la categoría social, de los niños en función del lazo entablado por sus progenitores. Los hijos ilegítimos eran niños concebidos por parejas que contrariaban el ideal normativo de familia de la época, basado en el matrimonio indisoluble. Más tarde, la oficina demográfica de Carrasco se ocupó de calcular tasas de ilegitimidad (proporción de nacimientos ilegítimos por cada mil nacimientos totales) y clasificarlos según la nacionalidad de los padres. ${ }^{27} \mathrm{Si}$ bien tuvo dificultades para alcanzar una cobertura nacional, Carrasco estableció una jerarquía entre las provincias registradas a partir de un ranking que las ordenaba según cifras crecientes de ilegitimidad (Argentina, 1902, p.87). Los Anuarios demográficos de la doctora Zauchinger continuaron la línea de Carrasco y de este modo consolidaron estadísticamente los supuestos que mantenían nupcialidad y natalidad asociadas, la primera como condición de la segunda. Para 1930, esta oficina seguía clasificando los nacimientos según su legitimidad, mientras que la oficina municipal desautorizaba la validez de este criterio, al borrar esa distinción de su grilla estadística. En este momento, la cuestión de los hijos ilegítimos estaba siendo reconsiderada en los ámbitos legislativos, los foros profesionales y la jurisprudencia (Cosse, 2007).

La salud de la población infantil se había convertido en foco de interés en un contexto de difusión de las preocupaciones en torno a la cantidad y la calidad de la población futura de la Nación, factores sobre los que se depositaba la conformación de una 'nueva raza argentina', expresión de la identidad nacional. Una serie de discusiones alrededor del tema de la mortalidad infantil surgieron al inicio del siglo XX. Estos debates incluyeron la cuestión de si en el país esas cifras eran elevadas o despreciables. ${ }^{28}$ Sin embargo, no existía aún un consenso sólido respecto de qué considerar como infante en términos estadísticos. En 1909, el doctor Enrique Foster - médico del Hospital de Niños y funcionario de la Administración Sanitaria y Asistencia Pública - publicaba en los Anales de esta institución un estudio que pretendía zanjar esta controversia, desautorizando los argumentos de quienes se equivocaban en "la manera de encarar las estadísticas" y tomaban sólo el primer año de vida para el cálculo de la mortalidad infantil (Foster, 1909, p.211). El primer Congreso Nacional de Medicina de 1916 lo respaldó, estableciendo como criterio uniforme el período de cero a dos años. Por su parte, en los estudios de la SDGM, Zauchinger sostenía un criterio amplio de la infancia que llegaba hasta la pubertad. Identificaba en este grupo social a las personas de cero a 15 años; acorde, en términos generales, con el concepto legalista mantenido por la cúpula del DNH que reconocía el límite de la infancia en lo determinado por la ley civil argentina (12 años para la mujer, 14 para el varón). ${ }^{29}$

La recopilación de las cifras de la mortalidad infantil le permitió a Zauchinger hacer un análisis de las causas de defunción de los niños y llegar a la conclusión de que "la mayor parte de las enfermedades pertenecen a la categoría de las evitables, susceptibles de ser influenciadas por la higiene y una profilaxis eficazmente dirigida" (Argentina, 1934, p.21). En esto se alineaba con los profesionales inclinados a la medicina social que, en el marco 
de los difundidos temores por la denatalidad y la necesidad de reforzar los factores endógenos de crecimiento demográfico - propios de la época de entreguerras - venían promoviendo iniciativas para estimular la procreación, combatir la mortalidad infantil y proteger a las madres y a sus hijos. Esta posición ya había sido expuesta por Zauchinger en las conclusiones de su tesis para concluir sus estudios médicos donde le proponía al Congreso Nacional reglamentar el trabajo de la mujer embarazada, establecer un seguro obligatorio para las obreras (garantizado por el Estado) y proteger a la primera infancia en general, pero especialmente a los niños ilegítimos, puesto que la doctora consideraba que las mayores tasas de mortalidad infantil estaban asociadas a los lugares con índices altos de ilegitimidad. Esta propuesta de tutela de la maternidad - formulada en 1910 - alcanzaba ahora mayor resonancia en un escenario donde la cuestión del potencial demográfico de la Nación se veía jerarquizada en la agenda política. Ni el ingreso de extranjeros ni la baja de los índices de mortalidad lograban compensar la caída de la natalidad. La disminución del crecimiento vegetativo alimentaba proyecciones pesimistas respecto del futuro de la 'raza argentina'; en ellas se justificaba la intervención médica dirigida a estimular el crecimiento poblacional.

Por otro lado, en una etapa en la que se encontraba interrumpida la edición del Anuario demográfico, ciertas enfermedades fueron objeto de trabajos especiales presentados por la SDGM. Las incidencias de la gripe, en ocasión de la epidemia de 1918/1919, y de la tuberculosis (en 1921) fueron analizadas en extensos cuadros, gráficos y mapas. Frente a la debilidad de esta agencia estadística, la denuncia obligatoria de ciertas enfermedades buscó favorecer la creación y/o ampliación de algunos de sus registros. En 1936, el Congreso Nacional promulgó la ley nacional 12.317 que hacía obligatoria la declaración de casos comprobados o sospechosos de enfermedades contagiosas y transmisibles. La norma constreñía a los médicos a denunciar ante las autoridades públicas locales los casos observados en consulta que se encuadraban en una lista de 28 enfermedades y a comunicarlos al DNH con mayor o menor celeridad según la enfermedad. ${ }^{30} \mathrm{Si}$ bien procuraba identificar focos epidémicos a fin de movilizar recursos y medidas profilácticas de emergencia, según uno de sus comentaristas, la ley también tenía como propósito fortalecer las estadísticas de morbilidad de cada región del país, de manera de aportar elementos de juicio a las decisiones públicas frente a los problemas sanitarios (Alvarado, 1937, p.227). La norma generaba una situación compleja: su cumplimiento dependía de la cooperación de los médicos a la vez que ponía en tensión el derecho galénico al secreto profesional. Pero, este tipo de normativa respondía a la propagación de la medicina social, a la problematización pública de las cuestiones demográficas y a la búsqueda del 'mejoramiento' médico y social de la población. Al mismo tiempo, pretendía impulsar ciertos avances en materia de producción estadística. Para una agencia pública con escasa capacidad de recolección como la SDGM, esta normativa procuraba resolver, 'desde afuera', muchos de sus impedimentos o limitaciones.

La estadística sanitaria oficial se encargó también de la publicación de algunas cifras basadas en registros administrativos de hospitales, que circularon en periódicos y revistas especializadas de medicina. ${ }^{31}$ Se trataba de la objetivación numérica de todo un dispositivo de atención y asistencia, controlado por los médicos, que se estaba poniendo en funcionamiento. En paralelo, las cifras del Boletín de Estadística Municipal condensaban la 
labor de la cada vez más densa red institucional de asistencia social de la Capital Federal. ${ }^{32}$ Incluso, agencias particulares de este entramado impulsaban sus propias publicaciones especializadas, como el Boletín Gráfico Estadístico de la Administración Sanitaria y Asistencia Pública (1925), capitalizando las ventajas que, para la divulgación general, acarreaba la técnica gráfica. ${ }^{33}$

A partir de la década de 1920, las denominadas enfermedades modernas (como las cardiovasculares y el cáncer) comenzaron a pasar a un primer plano en la escena pública, gracias a la visualidad que le brindaban las estadísticas. Enfermedades como las gastrointestinales, la sífilis, el paludismo y la tuberculosis se convirtieron en eje de las nuevas estrategias de la medicina social que empezaban a desplazar a la 'antigua' higiene defensiva (Armus, Belmartino, 2001; Armus, 2000a). Sin embargo, los acentos e insistencias del discurso de la profilaxis no se anclaban necesariamente en lo objetivado por las estadísticas, sino más bien se desentendían de ellas (Armus, Belmartino, 2001). En esos años, los insumos de información de la SDGM eran muy cuestionados. Esta agencia estatal no lograba una cobertura total del país, no podía garantizar uniformidad en la clasificación nosológica, ni asegurar que la certificación de las defunciones en muchas localidades del interior fuera hecha por un médico. ${ }^{34}$ La escasez y la falta de sistematicidad de las estadísticas vitales se pondrían más tarde en el foco de las demandas por un fundamento 'racional' de la política sanitaria argentina, ya en la antesala de los gobiernos peronistas. ${ }^{35}$ Por el otro, se trataba de una clase de matemáticas que a los médicos les resultaba algo desconocida. El lenguaje numérico tampoco estaba establecido firmemente como un criterio de autoridad legítimo en el campo.

\section{La atención volcada a la formación de cuadros técnicos}

Cuando Coni se graduó, y mientras se desempeñó en la oficina técnica estatal, la formación del médico profesional en la Argentina no se caracterizaba por prestar atención a las competencias estadísticas. El plan de estudio de la carrera de medicina, definido en 1874 por la Universidad de Buenos Aires (UBA), signó el modelo 'tradicional' de estudio de la disciplina que permaneció durante muchos años en distintas facultades del país, ya que dicho plan marcaba la orientación de las demás universidades que fueron incorporando la carrera. ${ }^{36}$ Recién tras la reforma discutida entre septiembre y octubre de 1899, la currícula de la UBA introdujo la epidemiología como asignatura, pero no por mucho tiempo. Las sucesivas reformas del plan de estudio - realizadas durante la primera mitad del siglo XX $(1919,1937,1948)$ - la dejarían fuera del perfil curricular. ${ }^{37}$ Sin embargo, el período en el que se dictó cierto contenido estadístico en la carrera (1900-1919) habría alcanzado para darle las herramientas cuantitativas básicas a quien continuó, décadas después, el legado de Coni en un espacio oficial.

Durante el paso de Zauchinger por la Facultad de Medicina de la UBA, el plan de estudios (reformado en 1907) contemplaba el dictado de una materia denominada clínica epidemiológica. En julio de 1900, el doctor José Penna había sido nombrado como su catedrático titular (Cantón, 1921). Se trataba de una asignatura del último año de una carrera que se estipulaba duraba siete años (que luego pasó a seis años). 
Para la década de 1920, no sólo la Facultad había dejado de formar en epidemiología sino que el Estado no había logrado capitalizar esa corta experiencia de enseñanza incorporando y/o reteniendo los cuadros más competentes en el uso de la herramienta. Las tareas estadísticas continuaban confiadas a empleados subalternos sin formación científica. Un observador de la época señalaba que la estadística demográfica y sanitaria argentina "no se realiza[ba] por gentes expertas, versadas en análisis matemático y en cálculo de las probabilidades; y no [podían] brindar entonces los resultados que era de esperar". La escasa formación del personal hacía que la labor estadística quedara reducida "al cálculo de una simple porcentual, a la mecánica primitiva de sumas y restas" (Delfino, 1925, p.1687). En ocasiones, junto a señalamientos como éste, se exigía el establecimiento de programas de selección del personal en las oficinas públicas.

Para la década de 1930, la estadística no era una herramienta valorada por las facultades argentinas en las que se dictaba la carrera de medicina. En esas casas de estudio no se manejaban los últimos progresos en la materia. La metodología estadística tampoco era incorporada como un instrumento científico necesario para enfrentar dificultades inherentes a la salud pública o para organizar los servicios de asistencia hospitalaria que entonces encaraban problemas de demanda insatisfecha - por falta de camas, escasez de recursos, etc. (Armus, Belmartino, 2001, p.287). Las estadísticas hospitalarias habían sido materia de discusión del Primer Congreso Nacional de Estadística realizado, por iniciativa oficial, en Córdoba en 1925. Aunque tuvieron un lugar secundario, algunos médicos participaron de esas discusiones, así como en la sección demográfica del Congreso. Por otra parte, reuniones técnicas del campo disciplinar empezaban a mostrar comunicaciones pobladas de estadísticas (como es el caso del Tercer Congreso Nacional de Medicina de 1926 o de algunas conferencias sobre estadísticas dictadas en el Círculo Médico de Córdoba o en la Asociación Médica Argentina).

Sin embargo, la aplicación de esta herramienta no estaba expandida entre la comunidad médica. Si bien el lenguaje estadístico era cada vez más utilizado en la literatura científica, los médicos, en general, no manejaban este léxico. Las características de las narrativas epidemiológicas que empezaron a aparecer - como las explicaciones del lento descenso de la mortalidad por tuberculosis, la enfermedad que más concitó la atención de los médicos argentinos durante los años 1930 - nos brindan un indicio al respecto. Según Armus y Belmartino (2001, p.297), algunas de estas narrativas eran "razonables", otras "tentativas", pero otras directamente "arbitrarias" y no alcanzaban a despejar la incertidumbre que rodeaba el saber en torno a esta enfermedad. La falta de 'criterio estadístico' entre los médicos era resaltada también por los contemporáneos. Para un observador autorizado del campo disciplinar, era necesario que la Universidad se volcara a capacitar al profesional para el ejercicio de la función sanitaria. Una formación especializada se había convertido en "una exigencia de la Administración Pública" (Piñero, 1940, p.684). Esa capacitación incluía el entrenamiento en la aplicación de las herramientas estadísticas; por eso revitalizaba la propuesta de incorporar la estadística en el plan de estudio de la carrera de medicina, bajo la idea de brindar cursos especiales en la formación de grado. 


\section{Polémicas en el campo disciplinar}

Si bien, durante el período estudiado, las estadísticas se fueron abriendo camino en la opinión del cuerpo médico, el consenso sobre el valor de la estadística en este campo específico de saber e intervención - como en cualquier otro - no fue automático sino producto de una construcción histórica. Al interior de esta comunidad profesional se presentaron discusiones en torno a los aspectos positivos y negativos de la herramienta cuantitativa. No todos dentro del grupo profesional aceptaron sin más la supuesta supremacía del 'nuevo' método numérico sobre el 'antiguo' método de la inducción. Por otra parte, los reclamos de algunos médicos a la estadística pública, respecto de sus alcances y la manera en que estas cifras estaban siendo producidas por una burocracia estatal cuya idoneidad era puesta en duda, agregaban cierto aditivo a la polémica. Aunque en este caso se partía del reconocimiento de la utilidad de las estadísticas en el campo de la medicina, sus objeciones de algún modo debilitaban o ponían en tensión la posición de quienes promovían ampliar el rol de los números públicos en este ámbito.

Entre los defensores de esta última posición se encontraba el doctor Víctor Delfino, quien integraba importantes organizaciones médicas de la época, editaba el prestigioso semanario La Semana Médica y comulgaba con los postulados de eugenismo. Esta doctrina, que proponía el mejoramiento de la población a través de la promoción de los agentes en condiciones de perfeccionar la 'calidad racial' y mediante la prevención de la reproducción de aquellos considerados nocivos (es decir, el control de los elementos 'disgénicos' de la sociedad) tuvo una importante influencia en el pensamiento y la práctica médica en el país. ${ }^{38}$ En su comunicación al Congreso Nacional de Estadística de 1925, Delfino opinaba que "no se conoce bien un fenómeno ni puede beneficiarse de las consecuencias que de su conocimiento derivan, si no se somete al cálculo y se trata de reducirle a los términos de una fórmula más o menos simple ... según la naturaleza del hecho indagado" (Delfino, 1925, p.1687). La capacidad del número de expresar de manera simplificada 'la verdad' era uno de los elementos resaltados por las argumentaciones a favor de la herramienta cuantitativa en los círculos médicos. También se destacaban las posibilidades de generalización y la 'objetividad' que le eran atribuidas al instrumento; la potencialidad de la estadística como una forma de condensar los aspectos estáticos y dinámicos, absolutos y relativos, de los problemas concernientes a la salud pública; la posibilidad del registro seriado, continuo y homogéneo, valorado como base de medidas preventivas; la fertilidad de índices de tipo predictivos que pudieran nutrir a las políticas sociales. Los médicos que compartían la valoración positiva de la herramienta pertenecían a un arco ideológico muy amplio, desde conservadores a socialistas y anarquistas. Ante los baches de la estadística oficial, algunos de ellos, como el médico anarquista Juan Lazarte, llegaron a encarar investigaciones personales y a crear registros propios, en general circunscriptos a espacios geográficos locales.

Para los opositores al 'numerismo' y la 'apariencia de exactitud', por el contrario, la estadística ahogaba la inteligencia médica; "hac[ía] del médico un agente contador, servidor pasivo de la cifra". En 1925, otra perspectiva era difundida por las páginas de la Revista del Círculo Médico Argentino: "Yo no reprocho al método numérico el hecho de contar, pues no se puede sistematizar sin contar; pero sí reprocho contar solamente, en una palabra atenerse 
al resultado riguroso como el matemático. Yo le reprocho contar demasiado, contar siempre, no querer poner espíritu en las cosas" (El método, 1925, p.1083). Para sus detractores, la estadística no era un método que por sí mismo condujera a la verdad, puesto que esa verdad yacía en "la apreciación del espíritu que juzga"; es decir, en el médico. Desde este punto de vista, la campaña a favor de la estadística se identificaba como un "charlatanismo de exactitud y verdad" que pretendía "engañar" a los menos experimentados (p.1085).

Las resistencias de los médicos al 'numerismo' deben inscribirse en la tensión que su presencia provocaba en la propia práctica médica. La enfermedad y la técnica de tratamiento guiado por el médico, se ubicaban en el registro de la singularidad: individualidad del paciente, pero también del acontecimiento único del encuentro médico-paciente, del diagnóstico y de la terapéutica. Sin embargo, la estadística procuraba una forma de categorización, estandarización y totalización ajena a esta tradición profesional. La categoría genérica, a través de la cual la estadística aprehendía al enfermo, ahogaba la especificidad del 'caso' como recorte individual. La renuencia de los médicos a la cuantificación restablecía, en primer plano, la concepción de que no hay enfermedades - a tratar de manera global y colectiva - sino enfermos. A su vez, la clasificación de enfermedades realizada por la estadística, la evaluación de tratamientos según estándares, la búsqueda de las relaciones constantes entre enfermedades y técnicas terapéuticas, devaluaban la intuición fundada en la experiencia personal del profesional. Por el contrario, su labor quedaba "reducid[a] a tomar un término medio que no será verdadero mañana, recurrir a esas fórmulas odiosas y datos ... [con] que se quier[e] bautizar [el] lenguaje médico: algunas veces, a menudo, generalmente" (El método, 1925, p.1085).

De allí la necesidad, según sus críticos, de recuperar el 'espíritu', la 'independencia' de criterio, el sello individual de ese 'arte de curar' afirmado sobre un conocimiento oficialmente acreditado, pero también - y fundamentalmente - apoyado en el instinto médico, el llamado ojo clínico del diplomado. Por el contrario, "el método numérico reconoce la potencia soberana de la cifra. El médico debe impedir los desbordes de su imaginación; él analiza, cuenta y registra severamente los resultados: nada más, nada menos" (El método, 1925, p.1082). Parecería que la imposición del criterio estadístico se hacía sentir como una limitación al libre ejercicio de una profesión que contaba en el país con una tradición marcadamente individualista. Posiblemente, al nivel del imaginario que rodeaba el campo médico, recaía sobre la herramienta cuantitativa la sospecha de poder volverse un instrumento de control externo sobre la práctica privada de la medicina. ${ }^{39}$ Así, los reclamos al Estado por más estadísticas desde algunas voces de la comunidad médica habrían entrado en contradicción con otra parte de ese grupo profesional que no estaba dispuesta a poner en riesgo la autonomía de sus espacios de ejercicio profesional.

Desde el punto de vista de quienes estaban a favor del método numérico, era necesario que las leyes de los grandes números desplazaran definitivamente conclusiones basadas en casos 'iluminadores', experiencias únicas de las que se desprendía un razonamiento inductivo. A su entender, la inducción debía quedar subordinada a la razón estadística. Como herramienta técnica, la estadística venía a sumar al campo médico un recurso de autoridad revalorizado en momentos en que los médicos diplomados argentinos competían todavía con la medicina casera o popular, las prácticas de automedicación y los curanderos 
(Armus, Belmartino, 2001, p.305). La incorporación de la objetivación estadística como principio de evidencia científico se mostraba en oposición al prejuicio y la superstición que - desde el punto de vista de los médicos profesionales - caracterizaban a esas otras formas de curar que disputaban su pretendido monopolio práctico y cognitivo. Además de postularse como un principio de legitimidad de la medicina acreditada, la estadística venía a funcionar como una nueva barrera de inclusión/exclusión del campo médico. Mientras el médico basaba su práctica en la precisión y las certezas de la estadística moderna, el quehacer del curandero - ejemplo de primitivismo e irracionalidad - descansaba en la mera creencia o en la superstición. De esta manera, la incorporación de la estadística en este campo del saber se traducía como el triunfo de la racionalidad (y las posibilidades de previsión) en el ámbito de la salud frente a las fuerzas del oscurantismo.

Sin embargo, paralelamente, la estadística demográfica y sanitaria argentina estaba siendo cada vez más criticada. A los cuestionamientos por los escasos progresos realizados en materia demográfica, se sumaban los reclamos por la falta de personal especializado, o al menos idóneo, en la dependencia pública encargada de producirla. La desconfianza hacia esos números oficiales parece haberse contagiado de la connotación negativa que recaía sobre la burocracia pública en general y que hegemonizaba el sentido común. En este sentido se manifestaba un observador contemporáneo: "casi todas las estadísticas hechas en el país, principalmente las médicas, son fraguadas o simplemente aproximadas (paludismo, lepra, tuberculosis, etc.), lo que resulta ... de la falta de método al hacerlas y de la más o menos grande ignorancia de los encargados de expurgarlas" (Delfino, 1925, p.1687). Las críticas hechas públicas mostraban el hiato que separaba la práctica real de elaboración de estadísticas del modelo científico imaginado y valorado para esa tarea. Aún así, desde el punto de vista de sus promotores, las tablas estadísticas, por más defectuosas que fueran, debían imponerse a "la buena voluntad y apreciación sentimental de hechos epidemiológicos ... en un país civilizado" (Alvarado, 1937, p.227).

La debilidad de la estadística demográfica no se agotaba en la escasa preparación de los funcionarios estatales. Como reconocía el propio DNH, una de las cuestiones básicas en las que arraigaba la deficiencia de sus estadísticas era la mala clasificación nosológica, debida a la ausencia de médicos en muchas localidades del interior que certificaran las defunciones o por la determinación errónea de las causas de muerte en los certificados extendidos por los profesionales. Muchas de las ambigüedades de la estadística nacional se remontaban, entonces, al primer eslabón de la cadena estadística y radicaban en la propia clasificación realizada por el médico. La calidad del dato estadístico se podía ver afectada por la impericia, negligencia u ocultación de parte del médico, así como por la incorrecta aplicación de la nomenclatura internacional adoptada en el país (Vaccaro, 1933, p.419). El rol clave del profesional en la producción de la estadística demográfica explica el pedido especial de cooperación al gremio médico que hiciera el DNH al fundar su repartición estadística (Argentina, 1911, p.5). El criterio y el compromiso del profesional con la labor estadística eran fundamentales. Sin embargo, este punto nodal escapaba a la mirada aguda y crítica de los profesionales de la medicina - poco comprometidos con los intereses estadísticos -, formados, por otra parte, en una escuela que desestimaba su importancia en el perfil profesional. 


\section{A modo de cierre}

En 1948, en el curso inaugural de médicos higienistas de la Escuela de Salubridad de la Universidad del Litoral, el profesor español Juan Moroder Muerda (1949, p.202) afirmaba: "Los odiados números, la estadística, enemiga natural del médico, ha venido a ocupar un puesto importante y sin ella los sanitaristas no sabemos ya andar por el mundo". Estas palabras expresan tanto las reticencias de este grupo profesional a las herramientas estadísticas como su creciente valoración. Así como había sido para los higienistas en la última parte del siglo XIX, a mediados de la centuria siguiente las estadísticas volvían a aparecer en el discurso de los médicos como un insumo insustituible de la acción sanitaria planificada. En el interludio, un contexto de creciente preocupación por las cuestiones relativas a la población - de controversias en torno a los factores de crecimiento demográfico, de interpretaciones cruzadas sobre las causas de la mortalidad infantil o de la caída de la natalidad, de disertaciones acerca de la 'calidad' del capital humano de la Nación - favoreció la divulgación de los números como códigos de lectura de esos problemas, aunque no redundó en una integración automática de la estadística como instrumento del saber médico. La salud de la población se había vuelto importante no sólo en términos de la ausencia de enfermedades sino, también, en función del 'vigor racial' y el futuro de la Nación. Para auscultar a esa entidad colectiva que constituía la Nación, y hacer el seguimiento de sus índices vitales, la comunidad médica argentina contó con un espacio de producción de información dentro del Estado, que se mantuvo durante décadas, aunque bajo la sombra de la crítica por sus escasos logros y la falta de preparación de su personal. La trayectoria de la SDGM mostró limitaciones a nivel organizativo, operativo y de recursos, así como dificultades para vencer obstáculos propios de otras reparticiones estatales - como los frenos a la centralización o la superposición de jurisdicciones. Estos factores excluyeron a la oficina demográfica de un lugar protagónico en la institucionalización de la estadística local.

Desde fines del siglo XIX, el lenguaje estadístico les posibilitó a los médicos intervenir en el debate público, racionalizar la gestión de la sociedad, ocupar un rol político. ${ }^{40}$ Para cuando los médicos buscaron afirmarse como los únicos proveedores de servicios de atención de la salud, y redoblaron esfuerzos frente a sus competidores - curanderos/as, homeópatas, farmacéuticos, herboristas, droguistas -, la estadística incorporó una nueva utilidad social: pasaba a ser una herramienta más en el complejo proceso de delimitación del campo específico. Sin embargo, lo que para algunos constituía un pilar adicional sobre el cual afirmar el monopolio de la práctica legítima de curar, por otros era visto con desconfianza y escepticismo por las derivaciones que el uso de estadísticas podía tener en la práctica profesional. Desde el punto de vista de los médicos que ocupaban posiciones en el Estado, la estadística fue valorada como un instrumento de unificación cognitiva del territorio en el marco de un proyecto de centralización político-administrativa de la atención sanitaria. Sin embargo, la fragilidad e impotencia de la repartición estatal a cargo de esas cifras le impidieron completar esa tarea.

Así, en el país, las estadísticas estuvieron lejos de cumplir el papel que le había sido otorgado por los propios médicos a fines del siglo XIX: ser el 'alma' racional de la nueva profilaxis, encauzar la política sanitaria por el camino de la observación metódica, seriada 
y homogénea, dotar a la moderna medicina social de una planificación orgánica y racional. Cuando el sistema de salud argentino empezó a mostrar claras señales de necesitar reformas, la autoridad técnica de esta herramienta - "enemiga natural del médico" - fue reclamada por la comunidad profesional. Se le pedía, entonces, suplantar razones de orden político, mitigar la influencia de intereses particulares y erradicar discrecionalidades propias de una gestión política partidaria de la salud. No se consideraba que con la 'razón estadística' de su lado la acción del poder público también ganaba una herramienta política.

\section{NOTAS}

* Una versión preliminar de este trabajo fue presentado en el Duodécimo Seminário Nacional de História da Ciência e da Tecnologia, Séptimo Congreso Latinoamericano de Historia de la Ciencia y la Tecnología, realizado en la Universidade Federal da Bahía, Brasil, entre el 12 y el 15 de noviembre de 2010. Agradezco los comentarios y las sugerencias del grupo del proyecto UBACyT "Trabajo y salud en la Argentina: saberes académicos y políticas", orientado por Karina Ramacciotti, así como a los miembros del Centro de Estudios sobre Saberes de Estado y Elites Estatales del Instituto de Desarrollo Económico y Social, dirigido por Mariano Plotkin. Sus discusiones durante estos últimos años nutrieron mi perspectiva de trabajo. Agradezco también a los evaluadores anónimos de la revista cuyas sugerencias contribuyeron a enriquecer el texto original.

${ }^{1}$ Como se puede observar, no se trata de una particularidad local sino de un movimiento de carácter regional. Hacia fines del siglo XIX, el panorama estadístico brasilero se encontraba dominado por un grupo de hombres de ciencia que contaban con tesis de doctorado en epidemiología o salud pública. Estos médicos se convirtieron en usuarios críticos de las cifras oficiales (Senra, 2007). En esta misma época, una figura concentraba la escena estadística local en México. El doctor Antonio Peñafiel fue fundador y presidente de la Dirección General de Estadística de ese país, creada en 1882 bajo la Secretaría de Fomento. Peñafiel también dirigió el primer Censo Nacional de población realizado en México en 1895, resultado de la presión ejercida por la corporación médica a través de sus organizaciones gremiales y colegiadas como la Academia Nacional de Medicina. El campo estadístico de ese país surgía en el entrecruzamiento del ámbito científico, el mercado - con el desarrollo de los seguros de vida - y el Estado, dados los lazos de los médicos con la elite política gobernante (Cházaro, 2010).

${ }^{2}$ Censo de la provincia de Buenos Aires, 1881; Censo Escolar de la República, 1883-1884; Censo General de la ciudad de Buenos Aires y de la provincia de Santa Fe, 1887; Censo Nacional Agropecuario, 1888; Censo de la provincia de Córdoba y de la de Buenos Aires, 1890.

${ }^{3}$ Según Armus y Belmartino (2001), desde el último tercio del siglo XIX y hasta 1916, se generó y afirmó en el Estado argentino una burocracia médico administrativa. Si bien no se trató de un grupo socialmente homogéneo, los higienistas trabajaban, entonces, en el Departamento Nacional de Higiene, en dependencias de gobiernos provinciales y en las asistencias públicas municipales. Su expansión burocrática alcanzó otras dependencias oficiales, no específicamente orientadas a cuestiones relativas a la salud, como el Consejo Nacional de Educación.

${ }^{4}$ La trayectoria social de este reconocido médico higienista es realmente rica. Incluye una prolífera labor de investigación, el desempeño profesional en hospitales y en distintas oficinas técnicas del Estado. Fue fundador de instituciones de salud y de asistencia social. Creó, dirigió y colaboró en diversas publicaciones (especializadas y no) y tuvo variadas participaciones en congresos internacionales. Resaltamos aquí en particular sus vínculos con la estadística pública. Para profundizar en su trayectoria, ver Álvarez, 2008 y su propio libro autobiográfico Memorias de un médico higienista (Coni, 1918).

${ }^{5}$ La función de Coni fue la única posición rentada de una comisión presidida por el doctor Diego G. De la Fuente, quien había dirigido el primer Censo Nacional en 1869.

${ }^{6}$ Por ejemplo, el doctor Coni, en funciones como delegado de la Oficina de Estadística de la provincia de Buenos Aires, publicó en 1878 varios trabajos sobre la viruela en Buenos Aires, uno con las cifras de muertes debidas a afecciones puerperales y otro muy importante sobre "La mortalidad de Buenos Aires entre 1869 y 1877". Sus trabajos sobre "El movimiento de la población de Buenos Aires desde su fundación hasta la fecha" y "La mortalidad infantil en Buenos Aires" datan de 1879 (Coni, 1918). Entonces, el gobierno de la provincia impulsaba la realización de publicaciones de pequeño formato en francés, alemán e italiano, orientadas a promover la inmigración y a estimular la radicación de capitales en el país. 


\begin{abstract}
${ }^{7}$ A la luz de las trayectorias sociales de los miembros de la generación posterior de expertos estadísticos, se observa que esa opacidad entre el carácter público de la actividad que desempeñaban y su capitalización privada permaneció durante la etapa de entreguerras (Daniel, 2010). Asimismo, es importante señalar que la superposición entre los ámbitos público y privado no fue exclusiva de la actividad estadística. Esta tensión está estrechamente ligada al proceso mismo de construcción del Estado argentino. Por tanto, atravesó también otras esferas de la administración pública en las que las reparticiones oficiales tuvieron que enfrentar las resistencias de instituciones de la sociedad civil que se venían ocupando de las materias sobre las que paulatinamente se fue expandiendo la 'estatalidad'. En el mismo campo de la salud, un ejemplo de ello radica en la experiencia estatal de asistencia social a la maternidad y la infancia y su relación problemática con las instituciones privadas, autárquicas o paraestatales preexistentes. Para profundizar, ver Biernat, Ramacciotti, 2008, p.334.
\end{abstract}

${ }^{8}$ La preocupación por la cantidad y la calidad de población futura de la Nación rodea esta advertencia. Desde el Segundo Congreso Médico Latinoamericano (1904) - presidido por el doctor Coni y en el que participó el titular de la oficina demográfica argentina, Gabriel Carrasco -, surgió una recomendación dirigida a estudiar un plan uniforme para los países americanos para levantar censos simultáneamente.

${ }^{9}$ Este censo fue presidido por Diego G. de la Fuente (1834-1909), secundado por Alberto Martínez (18681925) y Gabriel Carrasco (1854-1908).

${ }^{10} \mathrm{El}$ doctor Coni también participó en la organización de la sección demográfica del DNH en 1891, a pedido de su presidente el doctor Guillermo Udaondo; ejerció esta función de manera honorífica hasta 1893 (Coni, 1918, p.130-134).

${ }^{11}$ Alberto Martínez fue director de la Oficina de Estadística Municipal de la Ciudad de Buenos Aires hasta 1923. Inició su carrera estadística como vocal de la comisión del Censo Municipal de Buenos Aires en 1887. Unos años más tarde, se sumó a la comisión designada en 1890 por el intendente municipal para investigar el problema de la elevada mortalidad infantil en la ciudad de Buenos Aires; el doctor Emilio Coni era entonces presidente de esta comisión. Martínez participó en operativos censales de carácter provincial (Córdoba, 1890 y Mendoza, 1909) y fue parte de la comisión a cargo del Segundo Censo Nacional de población (1895). Como titular de la oficina de estadística, dirigió los censos generales de la ciudad capital de 1904 y 1909. En 1914, Martínez llegaría a ser presidente de la comisión del Tercer Censo Nacional de población. Para profundizar, ver Daniel, 2006; González Bollo, 2007.

${ }^{12}$ Obras de salubridad, tales como la instalación de cloacas, la construcción de redes de agua potable y de conductos pluviales, pero también la pavimentación de calles, las mejoras en la recolección de residuos y el control sanitario de alimentos, facilitaron el control de los ciclos epidémicos que tanto habían afectado a la ciudad de Buenos Aires en la segunda mitad del siglo XIX. Centrado en la salubridad y el equipamiento sanitario, el interés higiénico se depositó luego en la problemática de la pobreza y en la conformación de una red de instituciones de asistencia (Armus, 2000b).

${ }^{13}$ La Oficina Demográfica de Carrasco fue creada en 1899 como una forma de darle continuidad a la obra estadística del Segundo Censo Nacional de población (1895). Llegó a publicar 14 boletines demográficos en sus siete años de existencia (si bien empezó siendo una publicación trimestral, sus últimos números fueron anuales). Este abogado, nacido en Rosario, fue director del Primer Censo General de Santa Fe (1887) y participó en la comisión del Segundo Censo Nacional de población. Levantó censos electorales a pedido del ministro del interior, Joaquín V. González (Daniel, 2006).

${ }^{14}$ El MSA fue fundado el 23 de mayo de 1911 con el apoyo de instituciones académicas prestigiosas, como la Sociedad Científica Argentina, las Universidades de Buenos Aires y de La Plata, y de asociaciones corporativas como la Sociedad Rural Argentina. Con su creación, el ingeniero Tomás Amadeo se proponía cubrir la falta de instituciones no oficiales que se ocuparan de la cuestión social. El MSA se orientó al estudio de la realidad socioeconómica del país, pero también buscó intervenir en el conflicto social y en la definición de las políticas públicas. Con esta doble función - informativa y de mediación - la versión local del Museo Social integraba los perfiles de sus pares de Francia y Alemania. En 1915, durante su visita al país, el norteamericano Teodoro Roosvelt señaló que la función del MSA era la de "trabajar por la cirugía preventiva del cuerpo social" (El Museo..., 1915, p.1). La misión social preventiva del MSA tomaba cuerpo en acciones como el impulso a la creación de asociaciones mutuales y cooperativas obreras o la promoción de legislación social, entre otras.

${ }^{15}$ En el contexto de principios de siglo XX, la iniciación profesional de las mujeres en el campo médico exigía torcer resistencias y desafiar los roles socialmente establecidos. Todavía eran recientes las polémicas en torno al ingreso de la mujer a las escuelas médicas y los debates sobre sus 'capacidades' para la investigación científica. Adela Zauchinger tomó partido en estas discusiones con un artículo sobre la cuestión de la mujer médica que publicó en la Revista del Centro de Estudiantes de Medicina en 1903, recién ingresada en la carrera. 
${ }^{16}$ La doctora Zauchinger sobresale en el grupo de mujeres diplomadas en medicina de esta década que no se destacaron por ser grandes figuras públicas ni se caracterizaron por un fuerte compromiso social, como el grupo de pioneras de la generación anterior (en su mayoría militantes feministas).. En cuanto a su origen social, se trataba de mujeres que accedieron a la educación superior, en su mayoría provenientes de familias inmigrantes de clase media (Kohn Loncarica, Sanchez, 1996). Estas mujeres debieron abrirse paso en un campo dominado por hombres y alcanzar cierto reconocimiento.

${ }^{17}$ Durante su paso por la Facultad de Medicina, Zauchinger también conoció a quien, más tarde, sería la autoridad máxima del DNH, el pediatra y higienista doctor Gregorio Aráoz Alfaro. Él había sido su maestro y consejero, y formó parte del jurado evaluador de su tesis de grado.

${ }^{18}$ En su actuación profesional, las mujeres médicas entonces se desempeñaban principalmente en tocoginecología, pediatría, medicina social, enfermería; especialidades vistas como la traducción 'natural' de sus características de género y ligadas a su rol social de madres. Más allá del ejercicio privado de la profesión, se desempeñaron en instituciones públicas asociadas a la asistencia, hospitales, en el DNH o en el Consejo Nacional de Educación.

${ }^{19}$ A partir de que en 1917 se interrumpió el gasto para los corresponsales de las gobernaciones, sus datos se refirieron sólo a la Capital Federal y 14 provincias (período 1918-1929). Para profundizar en los obstáculos asociados a la recolección de información en los Territorios Nacionales, ver Di Liscia, 2009, p.262-263.

${ }^{20}$ Los datos acerca de la cantidad de personal de las distintas agencias de estadística fueron consultados en González Bollo, 2007.

${ }^{21}$ Emilio R. Coni dejó trascender en una importante revista del campo disciplinar - La Semana Médica su opinión favorable sobre la oficina demográfica, organizada por el doctor Penna, al considerar que había alcanzado un buen funcionamiento. En 1918, esa revista había celebrado el trabajo de su directora, la doctora Zaunchinger, "que ha hecho una especialidad de estas tareas, adquiriendo autoridad propia por su competencia y exactitud en los cómputos estadísticos” (Epidemiología, 1918, p.552).

${ }^{22}$ Al respecto, ver Argentina, 1926a. La Dirección General de Estadística Nacional realizaba cálculos post censales de población que publicaba anualmente. También procuró recabar información sobre nacimientos, matrimonios y defunciones de manera directa de los registros civiles de las gobernaciones, compitiendo claramente con la oficina de Zauchinger.

${ }^{23}$ La SDGM tuvo importantes dificultades para asegurar la regularidad de su producción estadística, mostrando importantes atrasos e interrupciones en sus publicaciones. La SDGM presentó los volúmenes del Anuario Demográfico, relativos a los años de 1911 a 1916, pero recién pudo retomar su publicación para los años 1930-1936 (volúmenes 7 al 10). Por otro lado, los datos de 1916, por ejemplo, no se alcanzaron a publicar hasta ocho años más tarde.

${ }^{24}$ Cabe, por supuesto, contemplar también que fuera exigua la relevancia de las tareas de recopilación estadística desde el punto de vista de quienes ocupaban altos puestos en la función pública y realizaban los nombramientos para este tipo de cargos. En este sentido, la escasa gravitación política de este ámbito lo habría vuelto pasible de una dirección femenina. Sin embargo, los sucesivos directores del DNH quienes nombraron primero a la doctora Zauchinger y luego la confirmaron en sus funciones reiteradas veces - reconocían su versación y competencia en la materia.

${ }^{25}$ Siri consideraba que en la Argentina se le venía dando escaso valor a las estadísticas demográficas como elementos de juicio y que esta constituía una de las principales fallas de los planes de organización de la atención médico-sanitaria y de asistencia social hasta el momento aplicados. En su opinión, la planificación debía fundamentarse en la realidad del país - muy diferente según las regiones - antes que priorizar los aspectos formales, como la división de tareas y jurisdicciones entre las diversas instituciones y niveles de gobierno que se ocupaban de la atención sanitaria. Con el objetivo de conocer en profundidad la situación y los problemas de salud inherentes a las distintas áreas del territorio, Siri creó en 1939 el Gabinete de Geografía Social. Procuraba estudiar las características y la distribución de la población en cada región a través de una encuesta (Biernat, Ramacciotti, 2008, p.339-340). Pero, al igual que la doctora Zauchinger, se chocaría con la reticencia de las provincias a entregar información.

${ }^{26}$ En las publicaciones de la doctora Zauchinger, las defunciones de los niños eran clasificadas por edad, nacionalidad y sexo. También diferenciaban si estas muertes eran provocadas por enfermedades gastrointestinales o infectocontagiosas.

${ }^{27}$ Retomaba así una distinción dentro del universo social de la infancia establecida por el Código Civil argentino en 1869. Según Cosse, la condición de hijos ilegítimos los hacía sujetos de una minusvalía jurídica, puesto que acarreaba una diferenciación de derechos. Para profundizar, ver Cosse, 2007. 
${ }^{28}$ El doctor Foster justificaba la amplitud de las repercusiones alcanzadas por el tema de la siguiente manera: "La mortalidad de la primera infancia es uno de los tópicos más interesantes por la cantidad de problemas que encierra, tanto higiénicos como sociológicos; no es de extrañar, pues, que sea tratado, a menudo, tanto por las revistas científicas como por la prensa diaria, de lo que debemos felicitarnos, pues ella es indispensable para facilitar la tarea de los que están encargados de estudiar esa mortalidad, buscar sus causas y tratar de combatirlas" (Foster, 1909, p.208).

${ }^{29}$ El sistema de clasificación utilizado por la doctora Zauchinger era el siguiente: 0 a 7 días, 7 a 30 días, 1 a 2 meses, 2 a 3 meses, 3 a 6 meses, 6 a 12 meses, 1 a 2 años, 2 a 6 años, 6 a 10 años, 10 a 15 años (Argentina, 1913). Es interesante señalar que su clasificación estadística priorizaba la etapa de la primera infancia ya que segmentaba en un número mayor de categorías (siete) a los menores de dos años, integrando al resto de la población infantil en sólo tres categorías que involucraban intervalos temporales más largos.

${ }^{30}$ La ley 12.317 establecía dos grupos. El primero reunía enfermedades como el cólera, la fiebre amarilla, la peste (bubónica, neumónica o septicémica), la viruela y el tifus exantemático. El grupo B estaba compuesto por enfermedades como la difteria, la escarlatina, el sarampión, el coqueluche, la fiebre tifoidea, la meningitis, la encefalitis, la poliomelitis, la tuberculosis, entre otras. El Ministerio del Interior se reservaba el derecho de extender la lista o suprimir algunas por decreto.

${ }^{31}$ Por ejemplo, la revista La Semana Médica se constituyó en un espacio de difusión de los anuarios estadísticos del DNH.

${ }^{32}$ En el apartado de salud pública de este boletín quedaban registrados: cantidades de enfermos asistidos, entradas y salidas de instituciones sanitarias (hospitales, manicomios, etc.) y asistenciales (asilos, casa de expósitos, patronato de la infancia), número de desinfecciones y de profilaxis preventiva.

${ }^{33}$ En esta etapa, sectores populares y clases medias urbanas se empezaron a empapar de esquemas y nociones científicas divulgadas por manuales y diarios de gran circulación en Buenos Aires. Para profundizar en la cuestión de la difusión de la 'imaginación técnica' ver Sarlo, 2004. Una primera aproximación al desarrollo de la gráfica estadística en la Argentina en Daniel, 2010.

${ }^{34}$ Este no era un punto menor en provincias donde, por carecer de médicos, la mortalidad sin causa justificada por falta de diagnóstico llegaba a más del 75\% o se ubicaba entre el 45\% y el 75\% (Argentina, 1926b).

${ }^{35}$ Para profundizar en estas discusiones, principalmente en los cuestionamientos del pediatra Florencio Escardó a la política hospitalaria desarrollada por Ramón Carrillo desde la Secretaría de Salud Pública, ver Ramacciotti, 2005.

${ }^{36}$ El modelo 'tradicional' de estudio de la medicina estuvo caracterizado por el enciclopedismo, la influencia de la medicina francesa y la falta de integración de contenidos o su desvinculación con el sistema asistencial. Este modelo se mantuvo en los cinco planes subsiguientes (1879, 1880, 1887, 1894, 1899). Para profundizar ver Arce, 1984.

${ }^{37}$ Recién en la década del 1960 se vuelven a ver cambios tan importantes en cuanto a la estructura de los planes de estudio de la carrera de medicina. Ellos formaron parte de un movimiento de renovación de la educación médica que atravesó toda América Latina. Con este cambio de paradigma, la Facultad de Medicina de la UBA adoptó un carácter más profesional, tomando como modelo a los Estados Unidos. En el plan de 1968 reapareció la materia Bioestadística y Epidemiología, pero en un lugar más devaluado: como una materia de la etapa inicial de la carrera que no exigía contar con ninguna materia previa. En 1972, la materia Bioestadística seguía formando parte del ciclo básico y pasaba a ser, junto a asignaturas como Medicina Sanitaria, Metodología Epidemiológica y Enfermería Médica, objeto de cursos cortos (con una carga horaria reducida de 36 horas).

${ }^{38} \mathrm{El}$ doctor Víctor Delfino fue un exponente de la organización institucional de la eugenesia que en la Argentina se produjo tras la Primera Guerra Mundial. Creó en 1918, la Sociedad Argentina de Eugenesia. Seis años antes había participado en el Primer Congreso Internacional de Eugenistas en Londres (1912) y tenía importantes conexiones con el movimiento eugénico internacional. Para profundizar en la propagación de ideas eugénicas en el país, ver Miranda, Vallejo, 2004, 2005; Palma, 2004; y sobre su difusión en América Latina ver Stepan, 1991.

${ }^{39}$ Según Desrosières (1996), en el dominio de la clínica médica, la resistencia de los médicos a los números se hizo más palmaria al momento en que se intentó establecer la comparación estadística como parámetro prioritario de la evaluación de la eficacia de diversas maneras de curar enfermedades.

${ }^{40}$ Para profundizar en la emergencia de la figura del médico-político, ver González Leandri, 2000. 


\section{REFERENCIAS}

ALVARADO, Carlos A.

La declaración obligatoria de las enfermedades contagiosas. Boletín Sanitario, Buenos Aires, año 1. 1937.

ÁLVAREZ, Adriana.

Tras la vida de un higienista y filántropo: Emilio Coni. In: Álvarez, Adriana. Saberes y prácticas médicas en la Argentina: un recorrido por historias de vida. Mar del Plata: Eudem. 2008.

ARCE, José.

La formación del médico en la República Argentina: análisis histórico del perfil curricular de la Facultad de Medicina desde su creación. Tesis (Doctorado) - Facultad de Medicina, Universidad de Buenos Aires, Buenos Aires. 1984.

ARGENTINA.

Ministerio del Interior. Departamento Nacional de Higiene. Oficina Demográfica. Anuario Demográfico 1930. Buenos Aires: Talleres Gráficos Ventriglia. 1934.

\section{ARGENTINA.}

Ministerio de Hacienda. Dirección General de Estadística Nacional. Informes, Buenos Aires, serie D, n.1. 1926a.

\section{ARGENTINA.}

Sección Demográfica del Departamento Nacional de Higiene. Consideraciones demográficas de la República Argentina, 1917, 1918, 1919. Memoria de la Sección Demográfica del Departamento Nacional de Higiene, Buenos Aires. 1926b.

\section{ARGENTINA.}

Ministerio del Interior. Departamento Nacional de Higiene. Oficina Demográfica. Anuario demográfico 1911. Buenos Aires: Talleres Gráficos de la Penitenciaría Nacional. 1913.

ARGENTINA.

Ministerio del Interior. Departamento Nacional de Higiene. Anales del Departamento Nacional de Higiene, Buenos Aires, v.18. 1912.

ARGENTINA.

Ministerio del Interior. Departamento Nacional de Higiene. Anales del Departamento Nacional de Higiene, Buenos Aires, v.17. 1911.

\section{ARGENTINA}

Ministerio del Interior. Oficina Demográfica Nacional. Boletín Demográfico Argentino, Buenos Aires, v.3, n.9. 1902.

ARMUS, Diego.

Consenso, conflicto y liderazgo en la lucha contra la tuberculosis, Buenos Aires, 1870-1950.
In: Suriano, Juan (Comp.). La cuestión social en la Argentina, 1870-1943. Buenos Aires: La Colmena. 2000a.

ARMUS, Diego.

El descubrimiento de la enfermedad como problema social. In: Lobato, Mirta Z. (Ed.). El progreso, la modernización y sus límites (18801916). Buenos Aires: Sudamericana. 2000b.

ARMUS, Diego.

Enfermedad, ambiente urbano e higiene social entre fines del siglo XIX y comienzos del XX. In: Armus, Diego (Comp.). Sectores populares y vida urbana. Buenos Aires: Clacso. 1984.

ARMUS, Diego; BELMARTINO, Susana. Enfermedades, médicos y cultura higiénica. In: Cattaruzza, Alejandro (Dir.). Crisis económica, avance del Estado e incertidumbre política (19301943). Buenos Aires: Sudamericana. 2001.

BIERNAT, Carolina; RAMACCIOTTI, Karina. La tutela estatal de la madre y el niño en la Argentina: estructuras administrativas, legislación y cuadros técnicos (1936-1955). História, Ciências, Saúde - Manguinhos. Rio de Janeiro, v.15, n.2, p.331-351. 2008.

CAMARGO, Alexandre de Paiva Rio. Números para o progresso: um panorama da actividade estatística na Primeira República. In: Instituto Brasileiro de Geografia e Estatística. Bulhões de Carvalho: um médico cuidando da estadística brasileira. Rio de Janeiro: IBGE. (Memória Institucional, 11). Disponible en: http://www.ibge.gov.br/home/presidencia/ noticias/pdf/bulhoes.pdf. Acceso en: 10 jan. 2012. 2007.

CANTÓN, Eliseo. Historia de la Facultad de Medicina y sus escuelas. Buenos Aires: Imprenta y Casa Editora Coni. 1921.

CHÁZARO, Laura.

La vida y su valor a discusión: juicios médicos y cálculos de probabilidades entre médicos mexicanos del siglo XIX. In: Senra, Nelson de Castro; Camargo, Alexandre de Paiva Rio (Org.) Estatísticas nas Américas: por uma agenda de estudos históricos comparados. Rio de Janeiro: IBGE. p.313-329. 2010.

CONI, Emilio R.

Memorias de un médico higienista. Buenos Aires: A. Flaiban. 1918.

COSSE, Isabella.

Ilegitimidades de origen y vulnerabilidad en la Argentina de mediados del siglo XX. Nuevo Mundo Mundos Nuevos. Debates. Disponible en: 
http://nuevomundo.revues.org/12502?lang=pt. Acceso em: 10 jan. 2012. 2007.

DANIEL, Claudia.

La sociedad (des)cifrada: configuraciones del discurso estadístico en Argentina (1890-1945). Tesis (Doctorado) - Facultad de Ciencias Sociales, Universidad de Buenos Aires, Buenos Aires. 2010.

DANIEL, Claudia.

El Estado argentino y sus estadísticas: el derrotero de un largo proceso de institucionalización (1864-1968). Illapa, Revista Latinoamericana de Ciencias Sociales, Lima, año 2, n.5, p.151-174. 2009.

DANIEL, Claudia.

El primer observatorio social argentino: Estado, censos y estadísticas oficiales (1869-1914). Tesis (Maestría) - Facultad de Ciencias Sociales, Universidad de Buenos Aires, Buenos Aires. 2006.

DELFINO, Víctor.

La estadística en la administración nacional. $\mathrm{La}$ Semana Médica, Buenos Aires, v.32, 2. sem., p.1687-1688. 1925.

DESROSIÈRES, Alain.

La política de los grandes números: historia de la razón estadística. Barcelona: Melusina. 1996.

DI LISCIA, María Silvia.

Cifras y problemas: las estadísticas y la salud en los Territorios Nacionales (1880-1940). Salud Colectiva, Buenos Aires, v.2, n.5, p.259-278. 2009.

EL MÉTODO.

Revista del Círculo Médico Argentino y Centro de estudiantes de medicina, Buenos Aires, v.25, p.1082-1086. 1925.

EL MUSEO....

El Museo Social Argentino. Boletín del Museo Social Argentino, Buenos Aires, v.4, n.37-38, p.15. 1915.

\section{EPIDEMIOLOGÍA}

La Semana Médica, Buenos Aires, v.25, n.20, p.552-555. 1918.

FOSTER, Enrique.

La mortalidad de la primera infancia en la ciudad de Buenos Aires. Anales de la Administración Sanitaria y Asistencia Pública, Buenos Aires, v.4, p.208-211. 1909.

GONZÁLEZ BOLLO, Hernán. La estadística pública y la expansión del Estado argentino: una historia social y política de una burocracia especializada (1869-1947). Tesis (Doctorado) - Universidad Torcuato Di Tella, Buenos Aires. 2007.
GONZÁLEZ LEANDRI, Ricardo.

El Consejo Nacional de Higiene y la consolidación de una elite profesional al servicio del Estado: Argentina, 1880-1900. Anuario de Estudios Americanos, Sevilla, v.61, n.2, p.571-593. Disponible en: http://

hdl.handle.net/10261/15078. Acceso en: 10 jan. 2012. 2004.

GONZÁLEZ LEANDRI, Ricardo.

Notas acerca de la profesionalización médica en Buenos Aires durante la segunda mitad del siglo XIX. In: Suriano, Juan (Comp.). $\mathrm{La}$ cuestión social en la Argentina, 1870-1943. Buenos Aires: La Colmena. p.217-243. 2000.

INDEC.

Instituto Nacional de Estadísticas y Censos. $\mathrm{La}$ actividad estadística en la República Argentina, 1550-1983. Buenos Aires: Indec. 1983.

KOHN LONCARICA, Alfredo; SÁNCHEZ, Norma Isabel.

La mujer en la medicina argentina - las médicas de la segunda década del siglo XX. Saber $y$ Tiempo, Buenos Aires, n.9, p.89-107. 2000.

KOHN LONCARICA, Alfredo; SÁNCHEZ, Norma Isabel.

La mujer en la medicina argentina: las médicas de la primera década del siglo XX. Saber y Tiempo, Buenos Aires, n.2, p.113-138. 1996.

LACEY, Michael; FURNER, Mary.

The State and social investigation in Britain and the United States. Cambridge: Cambridge University Press. 1993.

MASSÉ, Gladys.

Fuentes útiles para los estudios de la población argentina en el Instituto Nacional de Estadísticas y Censos: una visión histórica. Buenos Aires: Dirección de Estadísticas Poblacionales del Indec. 2000.

MAYER CELIS, Leticia.

Entre el infierno de una realidad y el cielo de un imaginario: estadística y comunidad científica en el México de la primera mitad del siglo XIX. México: El Colegio de México. 1999.

MENTZ, Raúl.

Sobre la historia de la estadística oficial argentina. Estadística Española, Madrid, v.33, n.128, p.501-532. 1991.

MIRANDA, Marisa; VALLEJO, Gustavo. La eugenesia y sus espacios institucionales en Argentina. In: Miranda, Marisa; Vallejo, Gustavo (Comp.). Darwinismo social y eugenesia en el mundo latino. Buenos Aires: Siglo XXI. p.145-192. 2005.

MIRANDA, Marisa; VALLEJO, Gustavo. Los saberes del poder: eugenesia y biotipología 
en la Argentina del siglo XX. Revista de Indias, Madrid, v.64, n.231, p.425-444. 2004.

MORODER MUERDA, Juan.

La administración sanitaria, confluencia y síntesis de las ciencias de la salubridad. Archivos de la Secretaría de Salud Pública, v.5, n.2, p.197212. 1949.

NOVICK, Susana.

Legislation about censuses and statistics in Argentina: 1854-1991. Trabalho apresentado al 13., World Congress of the International Economic History Association, 22-26 jul. 2002. Buenos Aires. 2002.

OFICINA DE ESTADÍSTICA...

Oficina de Estadística Municipal y Registro de Vecindad. Anexo: Defunciones de difteria durante los años 1884, 1885 y 1886 . Boletín Mensual de Estadística Municipal, Buenos Aires, v.1, n.7, p.33-40. 1887.

OTERO, Hernán.

Estadística y nación: una historia conceptual del pensamiento censal de la Argentina moderna, 1869-1914. Buenos Aires: Prometeo. 2006.

PALMA, Héctor.

La eugenesia en la Argentina. Saber y Tiempo, Buenos Aires, v.5, n.17, p.61-95. 2004.

PANTALEÓN, Jorge.

Una nación a medida. Buenos Aires: Al Margen. 2009.

PIÑERO García, Pedro.

La estadística en la orientación de la política sanitaria. La Prensa Médica, Buenos Aires, n.13, p.657-687. 1940.
RAMACCIOTTI, Karina.

Las voces que cuestionaron la política sanitaria del peronismo (1946-1949). In: Lvovich, Daniel; Suriano, Juan (Ed.). Las políticas sociales en perspectiva histórica: Argentina, 1870-1952. Buenos Aires: Prometeo. 2005.

SALVATORE, Ricardo.

Sobre el surgimiento del estado médico legal en la Argentina (1890-1940). Estudios Sociales, v.11, n.20, p.81-114. 2001.

SARLO, Beatriz.

La imaginación técnica: sueños modernos de la cultura argentina. Buenos Aires: Nueva Visión. 2004.

SENRA, Nelson de Castro.

Na Primeira República, Bulhões Carvalho legaliza a atividade estatística e a põe na ordem do Estado. Boletim do Museu Paraense Emilio Goeldi. Ciências Humanas, Belém, v.4, n.3, p.387-399. 2009.

SENRA, Nelson de Castro.

Historia de las estadísticas brasileras. Rio de Janeiro: IBGE. v.2. 2007.

STEPAN, Nancy.

The hour of eugenics: race, gender and nation in Latin America. London: Cornell University Press. 1991.

VACCARO, Juan M.

Organización de la estadística médica. Sociedad de Higiene y Microbiología, Buenos Aires, año 8, n.6, p.416-424. 1933.

\section{$\rightarrow \rightarrow \leftrightarrow<<$}

